\title{
Space-Time-Energy Equivalence
}

\author{
Vladimir Nikolaevich Sukhanov ${ }^{1}$
}

${ }^{1}$ Affiliation not available

December 22, 2021

\begin{abstract}
The equivalence of space-time and energy are the principle that everything that has space and time has an equivalent amount of energy, and vice versa. It is presented here as an axiom that has become widespread in physics and astrophysics.
\end{abstract}

\section{Hosted file}

51h_S_T_E_en.pdf available at https://authorea.com/users/451879/articles/550082-space-timeenergy-equivalence 